D

${ }^{1}$ Secretary of Assembly 3, European Respiratory Society.

2Lung Biology Group, Clinical Microbiology, Royal College of Surgeon in Ireland,

Education and Research Centre, Beaumont Hospital, Dublin, Ireland.

${ }^{3}$ Chair of Assembly 3, European Respiratory Society.

${ }^{4}$ Dept of Pulmonology, Leiden University Medical Center, Leiden, The Netherlands.

\title{
Assembly 3: Basic and Translational Sciences
}

\section{Meet the Assemblies}

Cite as: Greene CM, Hiemstra PS. Assembly 3: basic and translational sciences. Breathe 2018; 14: 67-68.
Assembly 3 is a dynamic and friendly Assembly. In 2017, the Assembly changed its name from "Cell and Molecular Biology" to its current name (Basic and Translational Sciences) to better reflect its activities and focus. We provide a platform for interaction between basic scientists and practising clinicians who are interested in the cellular and molecular mechanisms involved in healthy and diseased lungs. A key focus of our Assembly is tracking scientific developments that easily translate into better diagnostic technologies or enhanced patient care. As such we are centrally involved in devising the content of the Scientific Year in Review and Scientific Grand Round at the ERS International Congress.

There are currently 450 members of Assembly 3. We have a highly active and engaged long-range planning committee (LRPC) who work closely with the Assembly 3 officers and with the Assembly early career representative, Sabine Bartel. In addition to Sabine Bartel, another early career member is also a member of the LRPC. In fact, a large proportion of our membership, 212 in total, are under the age of 40 , and we actively encourage early career members to join our groups in order to actively participate in the various educational and scientific initiatives that we undertake each year. In addition to the postgraduate courses, skills workshops and meetthe-expert sessions that we coordinate at the ERS
International Congress, we also plan and organise a number of topical and cutting-edge translational science symposia, often in collaboration with colleagues from other Assemblies. For the ERS International Congress in 2017 we received 313 abstracts for the free communication sessions. Many of these were of very high quality and as a result $84 \%$ of these submissions were accepted and presented as oral or poster presentations in Milan last year.

Each year our members prepare and submit research seminar proposals and many of these have been selected to be awarded funding. Our most recent research seminar was held in February, 2018 in Berlin. This event brought together scientists and clinicians with a special interest in exploring the topic of "Cross-talk in the lung microenvironment: implications for understanding chronic lung disease". Our early career representative, Sabine Bartel, drafted the programme and co-chaired the research seminar, illustrating the active involvement of early career members in our Assembly. Research seminars are 2-3 day focused meetings that are an excellent opportunity for our members to immerse themselves within a specialist topic in an informal setting and liaise closely with leading experts in a field.

Another area where Assembly 3 plays a central role is in the ERS Lung Science Conference, 
where our members often serve as programme coordinators and member of the organising committee. This annual conference, held in March each year, is at the forefront of basic and translational respiratory science and it is an ideal event for early career respiratory researchers. It offers a unique opportunity to network with peers from across the globe and features cuttingedge presentations on novel experimental lung research. The topic of this year's 16th Lung Science Conference is "Cell-matrix interactions in lung disease and regeneration".

The ERS encourages its membership to submit proposals for task forces on key topics relevant to respiratory health and clinical practice. Assembly 3 is currently leading two task forces that are developing statements on "Optimising experimental research in respiratory disease" and "Electronic cigarettes".

Assembly 3 currently includes the following groups.

\section{Group 3.1 Molecular Pathology and Functional Genomics}

The objective of this group is to enhance understanding of important mechanisms in lung biology, including processes underpinning lung development and lung pathophysiology. Its organisation is dedicated to bringing together basic research and clinical investigations. Group 3.1 wishes to showcase new developments at both the cellular and molecular level, and to facilitate the dissemination of new approaches for the diagnosis, treatment and prevention of respiratory diseases. At the time of writing, Philippe Bonniaud and Aurélie Fabre are the chair and secretary of this group, respectively.

\section{Group 3.2 Airway Cell Biology and Immunopathology}

The current chair of group 3.2 is Silke Meiners, and Irene Heijink is the secretary. This group offers a platform for scientists studying the cellular and molecular biology of the respiratory tract in health, disease and development. A major focus of this group is to promote understanding of the roles that airway epithelial and inflammatory cells play in innate host defense. There is a special emphasis on mechanisms related to intracellular function (cell signalling, gene expression) and cell secretion (cytokines, antimicrobial peptides, oxidants and proteases), and how these can become altered in disease processes.

\section{Group 3.3 Mechanisms of Lung Injury and Repair}

Chaired by Melanie Koenigshoff, this group brings together members who are interested in the cellular and molecular basis of injury to the lungs and new approaches to treatment for lung injury. Agnes Boots, previously the Assembly early career representative, is group 3.3 secretary. Its members include clinicians, clinician investigators and basic scientists who share these common interests. Principal areas of focus for group 3.3 are the mechanisms of disease related to acute lung injury (e.g. acute respiratory distress syndrome), fibrotic reactions, injury created by host-pathogen interactions, drug-induced lung injury and other mechanisms of acute and chronic lung injury.

\section{Assembly 3 highlights}

- An active and lively assembly with many educational and scientific events at the ERS International Congress

- Very good representation of early career members

- Effective and collegial links with many other Assemblies

- Centrally involved in the Lung Science Conference, research seminars and task forces

\section{Conflict of interest}

C.M. Greene reports research grants from H2020 (MCSA-IF), Cystic Fibrosis Therapeutics and the Irish Research Council (PhD fellowship), outside the submitted work. P.S. Hiemstra reports research grants from Galapagos NV and Boehringer Ingelheim, outside the submitted work. 